\title{
THE EFFECTIVE CSR FORCES FOR AN ENERGY-CHIRPED BUNCH UNDER MAGNETIC COMPRESSION
}

\author{
R. Li, Jefferson Lab, 12000 Jefferson Ave., Newport News, VA 23606, USA
}

\section{INTRODUCTION}

Following our earlier formulation of the coherent synchrotron radiation (CSR) effect on bunch dynamics in magnetic bends, here we investigate the behavior of the effective CSR forces for an energy-chirped Gaussian bunch in the bending plane around full compression, with special care being taken in the incorporation of the retardation relation. Our results show clearly a delayed response of the CSR forces to the compression or lengthening of the bunch length. In addition, around full compression, our results reveal sensitivity of the effective CSR forces on the particles' transverse position, as a consequence of the geometry of particle interaction and retardation in this regime. These results can serve as benchmarks to the numerical simulation of the CSR effects.

\section{THE DYNAMICAL EQUATIONS}

Consider an electron bunch moving ultrarelativistically on a curved orbit in a bending system. For a submilimeter bunch with high charge, the curvature-induced particle acceleration often causes the beam to emit synchrotron radiation coherently, and meanwhile the particles' collective interaction is often dominated by the acceleration term in the Lienard-Wiechert fields. This is called the coherent synchrotron radiation (CSR) effect. From the canonical formulation of the CSR effect in magnetic bends using Hamiltonian [1], one obtains the first order equation of motion for the electrons

$$
\frac{d X}{d s}=Y(X, s) \equiv M(s) X+F^{[f]}(X, s)
$$

for $X^{T}=\left(x, x^{\prime}, y, y^{\prime}, z, \delta_{H}\right), \quad\left[F^{[f]}(X, s)\right]^{T}=$ $\left(0, \tilde{F}_{x}, 0, \tilde{F}_{y}, 0, \tilde{F}_{H}\right)$, and

$$
M(s)=\left(\begin{array}{cccccc}
0 & 1 & 0 & 0 & 0 & 0 \\
-k_{x}^{2}(s) & 0 & 0 & 0 & 0 & \kappa_{0}(s) \\
0 & 0 & 0 & 1 & 0 & 0 \\
0 & 0 & -k_{y}^{2}(s) & 0 & 0 & 0 \\
-\kappa_{0}(s) & 0 & 0 & 0 & 0 & 0 \\
0 & 0 & 0 & 0 & 0 & 0
\end{array}\right) .
$$

Here $M(s) X$ in Eq. (1) reflects the nominal linear optics, with the horizontal and vertical focusing strengths $k_{x}(s)$ and $k_{y}(x)$ due to external magnetic fields satisfying $k_{x}^{2}(s)=\kappa_{1}(s)-\kappa_{0}^{2}(s)$ and $k_{y}^{2}(s)=-\kappa_{1}(s)$, and $\kappa_{0}(s)=1 / R(s)$ for $R(s)$ being the radius of the curved orbit. The relative canonical energy offset is defined as

$$
\delta_{H} \simeq \frac{e \tilde{\Phi}+E}{\gamma_{0} m c^{2}}-1
$$

05 Beam Dynamics and Electromagnetic Fields
The term $F^{[f]}(X, s)$ contains the normalized effective CSR forces as expressed in terms of potentials:

$$
\left\{\begin{aligned}
\tilde{F}_{x}(X, s) & \simeq \kappa_{0}\left(\tilde{A}_{s}-\beta_{0} \tilde{\Phi}\right)+\frac{\partial\left(\tilde{A}_{s}-\beta_{0} \tilde{\Phi}\right)}{\partial x}-\frac{\partial \tilde{A}_{x}}{\partial s} \\
\tilde{F}_{y}(X, s) & \simeq \frac{\partial\left(\tilde{A}_{s}-\beta_{0} \tilde{\Phi}\right)}{\partial y} \\
\tilde{F}_{H}(X, s) & \simeq \frac{\partial\left(\tilde{A}_{s}-\beta_{0} \tilde{\Phi}\right)}{\partial z}
\end{aligned}\right.
$$

with the potentials depending on the bunch phase space distribution $f(X, s)$

$$
\begin{aligned}
& {\left[\tilde{A}_{s}-\beta_{0} \tilde{\Phi}\right](x, y, z, s) \simeq} \\
& \frac{2 \beta_{0} r_{e}}{\gamma_{0}} \int d s_{r} \int d X_{r} f\left(X_{r}, s_{r}\right)\left(\frac{-(\Delta s)^{2}}{2 R^{2}}\right) \delta(P) \theta[Q] \\
& \tilde{A}_{x}(x, y, z, s) \simeq \\
& \quad \frac{2 \beta_{0}^{2} r_{e}}{\gamma_{0}} \int d s_{r} \int d X_{r} f\left(X_{r}, s_{r}\right)\left(\frac{-\Delta s}{R}\right) \delta(P) \theta[Q]
\end{aligned}
$$

for

$P=\left[\left(s-s_{r}\right)-\left(z-z_{r}\right)\right]^{2}-\beta_{0}^{2}\left[\mathbf{r}(x, y, s)-\mathbf{r}\left(x_{r}, y_{r}, s_{r}\right)\right]^{2}$ $Q=\left(s-s_{r}\right)-\left(z-z_{r}\right)$,

with $r_{e}$ the electron radius, $X_{r}=\left(x_{r}, x_{r}^{\prime}, y_{r}, y_{r}^{\prime}, z_{r}, \delta_{H r}\right)$ representing the source particle's phase space variables at the retarded pathlength $s_{r}$, and $\mathbf{r}(x, y, s)$ and $\mathbf{r}\left(x_{r}, y_{r}, s_{r}\right)$ the position vectors for the test and source particles respectively. In Eq. (3), the Dirac $\delta$ function and the Heaviside step function $\theta(x)$ together imply that the source particle's retarded longitudinal position is determined by

$$
\begin{aligned}
z_{r} & =z_{r}\left(x, y, z, s ; x_{r}, y_{r}, s_{r}\right) \\
& \equiv z-s+s_{r}+\beta_{0}\left|\mathbf{r}(x, y, s)-\mathbf{r}\left(x_{r}, y_{r}, s_{r}\right)\right|
\end{aligned}
$$

which imposes the retardation relation

$$
t_{r}=t-\frac{\left|\mathbf{r}(x, y, s)-\mathbf{r}\left(x_{r}, y_{r}, s_{r}\right)\right|}{c}
$$

on the interaction between particles.

\section{THE EFFECTIVE CSR FORCES}

Next, we focus on the CSR interaction for a 2D Gaussian bunch in the bending plane on a circular orbit of constant radius $R_{0}$, with an initial linear energy chirp $\delta_{H 0}=\delta_{H 0}^{\text {un }}+$ $u z_{0}$. We will calculate the first order effective CSR forces for the bunch, assuming that the single particle dynamics is only influcenced by the linear design optics

$$
X(s)=\mathcal{R}(s) X_{0}(0)
$$


for $\mathcal{R}(s)=R(s) A^{-1}$, with

$$
R(s)=\left(\begin{array}{cccc}
R_{11}(s) & R_{12}(s) & 0 & R_{16}(s) \\
R_{21}(s) & R_{22}(s) & 0 & R_{26}(s) \\
R_{51}(s) & R_{52}(s) & 1 & R_{56}(s) \\
0 & 0 & 0 & 1
\end{array}\right)
$$

and

$$
A=\left(\begin{array}{cccc}
1 & 0 & 0 & 0 \\
\alpha_{x 0} / \beta_{x 0} & 1 & 0 & 0 \\
0 & 0 & 1 & 0 \\
0 & 0 & -u & 1
\end{array}\right)
$$

and $X_{0}=\left(x_{0}, x_{0}^{\prime}, z_{0}, \delta_{H 0}\right)$ is the normalized phase space parameters for the particle at $s=0$. Let us assume that the initial normalized phase space distribution $\rho\left(X_{r 0}, 0\right)$ takes the form

$$
\rho\left(X_{r 0}, 0\right)=N \rho_{\mathrm{un}}\left(Z_{r 0}\right) \lambda_{0}\left(z_{r 0}\right),
$$

with $N$ the total number of electrons, $\lambda_{0}\left(z_{r 0}\right)$ the initial longitudinal charge density distribution function

$$
\lambda_{0}\left(z_{0}\right)=\frac{1}{\sqrt{2 \pi} \sigma_{z 0}} \exp \left(-\frac{z_{0}^{2}}{2 \sigma_{z 0}^{2}}\right)
$$

and $\rho_{\text {un }}\left(Z_{r 0}\right)$ the initial intrinsic (or uncorrelated) distribution in $Z_{0}=\left(x_{0}, x_{0}^{\prime}, \delta_{H 0}\right)$ space, with transverse emittance $\epsilon_{x 0}$ and (canonical) energy spread $\sigma_{H 0}$,

$$
\begin{aligned}
\rho_{u n}\left(Z_{0}\right) & =\frac{1}{\sqrt{2 \pi} \sigma_{H 0}} \exp \left(-\frac{\delta_{H 0}^{2}}{2 \sigma_{H 0}^{2}}\right) \\
\times & \frac{1}{2 \pi \epsilon_{x 0}} \exp \left(-\frac{x_{0}^{2}}{2 \sigma_{x 0}^{2}}-\frac{x_{0}^{\prime 2}}{2 \sigma_{x 0^{\prime}}^{2}}\right)
\end{aligned}
$$

for $\sigma_{x 0}=\sqrt{\beta_{x 0} \epsilon_{x 0} / \gamma_{0}}$ and $\sigma_{x 0^{\prime}}=\sqrt{\epsilon_{x 0} / \gamma_{0} \beta_{x 0}}$, with $\beta_{x 0}$ the initial transverse beta function at $s=0$. We then apply Eq. (4) to Eqs. (2) and (3) by changing the variables from $X_{r}$ to $X_{r 0}=\mathcal{R}^{-1}(s) X_{r}$. The effective longitudinal force in Eq. (2), for a particle at $(x, z, s)$ on a circular orbit with radius $R_{0}$, is

$$
\begin{aligned}
\tilde{F}_{H}^{\left[f^{(0)}\right]} & (X, s)=\frac{\beta_{0}^{2} N r_{e}}{\gamma_{0}\left|R_{0}\right|} \\
& \times\left(\int_{\Omega^{(+)}} d s_{r} d Z_{r 0} g^{(+)}+\int_{\Omega^{(-)}} d s_{r} d Z_{r 0} g^{(-)}\right),
\end{aligned}
$$

where the integrand $g^{( \pm)}$in Eq. (5) is

$$
\begin{aligned}
& g^{( \pm)}=\frac{-(\Delta \bar{s})^{2}}{2\left|\mathcal{R}_{55}\left(s_{r}\right)\right| \sqrt{b^{2}-a c}} \\
& \times\left[\rho_{u n}\left(Z_{r 0}\right) \frac{\partial \lambda_{0}\left(z_{r 0}^{( \pm)}\right)}{\partial z_{r 0}}-u \lambda_{0}\left(z_{r 0}^{( \pm)}\right) \frac{\partial \rho_{u n}\left(Z_{r 0}\right)}{\partial\left(\delta_{H}\right)_{r 0}}\right]
\end{aligned}
$$

for $a, b, c$ listed in Ref. [1], $\Delta \bar{s}=\left(s-s_{r}\right) /\left|R_{0}\right|$, and $z_{r 0}^{( \pm)}$ and $\Delta z^{( \pm)}$defined by

$$
\begin{aligned}
z_{r 0}^{( \pm)}\left(x, z, s ; Z_{r 0}, s_{r}\right) & =\frac{z-\Delta z^{( \pm)}}{\mathcal{R}_{55}\left(s_{r}\right)} \\
& =\left|R_{0}\right| \frac{a \bar{z}-b \mp \sqrt{b^{2}-a c}}{a \mathcal{R}_{55}\left(s_{r}\right)} .
\end{aligned}
$$

In Eq. (5), the domains of integration $\Omega^{( \pm)}$are set so as to ensure the existence of solutions for $z_{r 0}^{( \pm)}$and to exclude the advanced solutions

$\Omega^{( \pm)}=\left\{b^{2}-a c \geq 0\right.$ and $\left.Q^{( \pm)}=\Delta s+z_{r 1}-\Delta z^{( \pm)} \geq 0\right\}$.

One can show [2] that for a source particle in a tilted bunch to emit EM fields from the pathlength $s_{r}$, which interacts with the test particle at $(x, z, s)$, the source particle is limited to a region of the initial phase space

$$
\eta \equiv \sum_{j=1,2,6} \alpha_{j}\left(s_{r}\right)\left(X_{r 0}\right)_{j} \leq \eta_{\min }
$$

with

$$
\alpha_{j}\left(s_{r}\right)=\frac{\chi_{r} \mathcal{R}_{1 j}\left(s_{r}\right)-\mathcal{R}_{5 j}\left(s_{r}\right)}{\left|R_{0}\right|}
$$

and

$$
\eta_{\min }=A-\sqrt{B}
$$

With notation

$$
\hat{x}=\frac{x}{R_{0}}, \bar{z}=\frac{z}{\mid R_{0}}, \chi_{r}=\frac{\mathcal{R}_{55}\left(s_{r}\right)}{\mathcal{R}_{15}\left(s_{r}\right)},
$$

$A$ and $B$ in Eq. (7) are defined as

$$
\begin{aligned}
& A=\Delta \bar{s}\left(1-\hat{\chi}_{r} \Delta \bar{s} / 2\right)+\left(\hat{\chi}_{r} \hat{x}-\bar{z}\right), \\
& B=\left(1-\chi_{r}^{2}\right) \Delta \bar{s}^{2}\left(1-\frac{\Delta \bar{s}^{2}}{3}-\frac{1}{\gamma_{0}^{2}}+2 \hat{x}\right) .
\end{aligned}
$$

One can further reduce the integral in Eq. (5) by changing the variables from $\left(x_{r 0}, x_{r 0}^{\prime}, \delta_{H r 0}\right)$ in Eq. (5) to $\left(q_{1}, q_{2}, q_{3}\right)$, with

$$
q_{3}=\frac{\left|R_{0}\right|}{\beta_{x 0}} \eta
$$

for $\eta$ in Eq. (6), and letting $q_{1}, q_{2}$ be orthorgonal to $q_{3}$. This allows the reduction of the integral in Eq. (5) to a 2D integal over $s_{r}$ and $q_{3}$

$$
\begin{aligned}
& \tilde{F}_{H}(x, z, s)=C_{0} \int_{-\infty}^{\infty} d \Delta \bar{s} \frac{\Delta \bar{s}^{2}}{\left|u R_{16}\left(s_{r}\right)\right| \sqrt{\operatorname{det} \Upsilon\left(s_{r}\right)}} \\
& \times \int_{-\infty}^{q_{\min }} \frac{d q_{3}}{p\left(s_{r}, q_{3}\right)}\left[I_{0}^{(+)}+I_{0}^{(-)}\right]
\end{aligned}
$$

for

$$
C_{0}=\frac{\beta_{0}^{2} N r_{e}}{\gamma_{0} \sigma_{z 0}^{2}},
$$

where $p\left(x, z, s, s_{r}, q_{3}\right), \Upsilon\left(s_{r}\right)$ and $I_{0}^{( \pm)}\left(x, z, s, s_{r}, q_{3}\right)$ are functions of $\mathcal{R}_{i j}\left(s_{r}\right)$ and the intrisic rms values of the initial bunch phase space parameters, as listed in Ref. [2].

For a thin bunch with mild tilt, the upper integration limit $q_{\text {min }}\left(x, z, s, s_{r}\right)$ in Eq. (8) covers the whole beam phase space distribution, so the integration range for $q_{3}$ is effectively $(-\infty, \infty)$. However, for a thick bunch around full compression, for each $s_{r}$, the upper limit $q_{\text {min }}$ selects a portion of the initial bunch phase space $Z_{r 0}$ to interact with the test particle at $(x, z, s)$. Such selection of the initial phase space is sensitive to the test particle's internal coordinates $(x, z)$ inside the bunch. This causes the sensitive dpendence of the effective CSR forces on the $(x, z)$ of the test particle. 


\section{AN EXAMPLE}

To illustrate the behavior of the effective longitudinal CSR force calculated from the above formula, here we devise a model chicane which consists of three rectangle dipole magnets with bending radius $R_{0}=1 \mathrm{~m}$. The length for the two side dipoles is $L_{b}=0.3 \mathrm{~m}$ and for the center dipole $L_{B}=0.6 \mathrm{~m}$. The drift length between ajacent dipoles is $L_{d}=0.4 \mathrm{~m}$. For the bunch entering the chicane, we assume a $\delta-z$ correlation $u=-10.56 \mathrm{~m}^{-1}$. The resulting bunch compression factor, $\mathcal{R}_{55}(s)=1+u R_{56}(s)$ vs. $s$, is plotted in Fig. 1. Note that the bunch reaches full compression at $s_{c}=1.2 \mathrm{~m}$ when $\mathcal{R}_{55}\left(s_{c}\right)=0$ or $\chi\left(s_{c}\right)=0$.

Assume the 2D Gaussian bunch has initial transverse twiss parameters $\beta_{x 0}=5 \mathrm{~m}, \alpha_{x 0}=1$, and $\epsilon_{x 0}=1 \mathrm{~mm}$ mrad. The initial longitudinal parameters of the bunch are $\sigma_{H 0}=10^{-4}, \sigma_{z 0}=0.5 \mathrm{~mm}$, and $E_{0}=70 \mathrm{MeV}$. The bunch is transported through the chicane following design optics, and the CSR forces generated by the bunch on test particles are calculated. For $s=1.1$ to $1.3 \mathrm{~m}$, the normalized effective longitudinal CSR force in Eq. (8), for $(x, z)$ around the bunch, is displayed by the gray mesh in Fig. 2 . In addition, we use $\mathrm{N}=1000$ particles to represent the Gaussian bunch distribution and propagate them through the design optics. At each pathlength, the $\left(x_{k}, z_{k}\right)$ coordinates of these particles $(k=1$ to $N)$ are shown as the green dots in Fig. 2; and the CSR force $F_{k}=F_{H}\left(x_{k}, z_{k}, s\right)$ ( normalized by $C_{0}$ in Eq. (9)) on these particles are plotted as the red dots in Fig. 2. The average of $F_{k}$ over these particles, $\langle F\rangle=(1 / N) \sum_{k} F_{k}$, which represents the strength of the CSR interaction, is shown as the solid red curve in Fig. 3, which is compared with the blue solid line representing $\langle F\rangle$ for a rigid-line bunch [3].

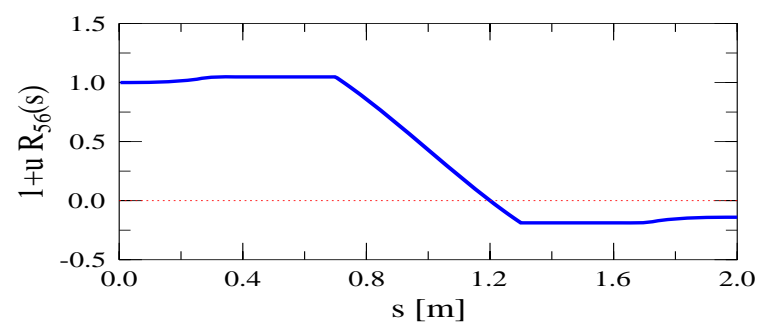

Figure 1: Compression factor $\mathcal{R}_{55}(s)=1+u R_{56}(s)$ vs. $s$ for the example chicane when $u=-10.56 \mathrm{~m}^{-1}$.

\section{DISCUSSIONS}

Our study of the CSR interaction for an energy-chirped 2D Gaussian bunch on a circular orbit shows that when the bunch is around full compression, the effective longitudinal CSR force on the bunch has a strong dependence on the particles' internal position inside the bunch. Besides, we also show that the strength of these forces has a delayed response to the variation of bunch length. Similar behaviors are also found for the transverse CSR forces. These analytical results can serve as benchmark to the CSR simulations. For example,the macroparticle model in our previous CSR simulation [4] was too crude to accurately model
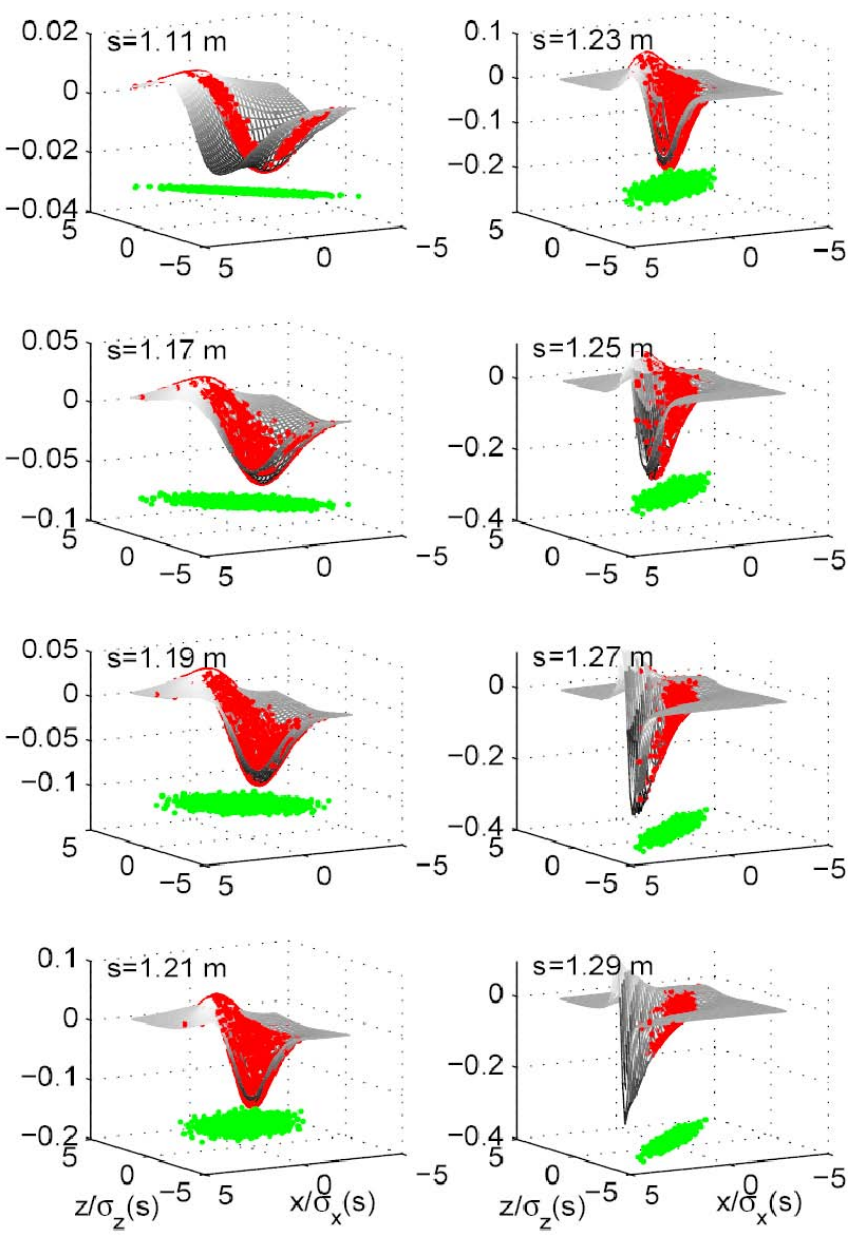

Figure 2: $F_{H}(x, z, s) / C_{0}$ vs. $x / \sigma_{x}(s)$ and $z / \sigma_{z}(s)$ at various pathlength $s$.

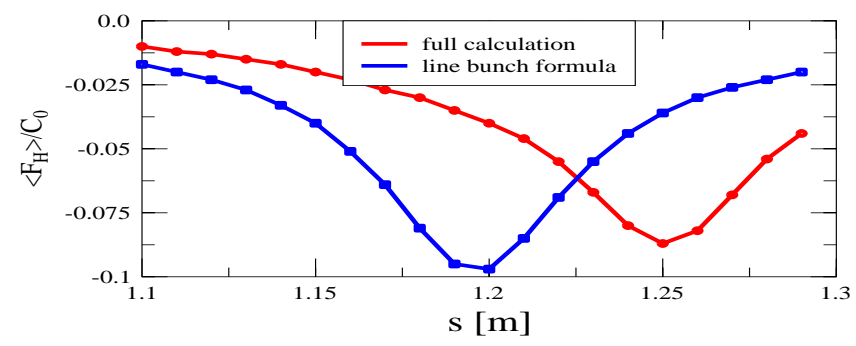

Figure 3: Mean of $F_{H} / C_{0}$ for the 1000 test particles vs $s$.

the detailed sensitivity of the effective CSR forces around full compression, and thus improvement of the simulation model is required.

This work was supported by U.S. DOE under Contract No. DE-AC05-06OR23177.

\section{REFERENCES}

[1] R. Li, JLAB-TN-05-046, 2005.

[2] R. Li, to be published.

[3] Y. S. Derbenev, J. Rossbach, E. L. Saldin, and V. D. Shiltsev, DESY Report No. TESLA-FEL-95-05, 1995.

[4] R. Li, Nucl. Instrum. Meth. Phys. Res. A 429, 310 (1998). 